\title{
Clinico-Serological Profile and Geographical Distribution of Dengue Fever from a Tertiary Care Hospital, South India
}

\author{
Morubagal Raghavendra Rao (iD), Rashmi Padmanabha Mahale (iD, \\ Sowmya Shivappa (D), Vidyavathi B. Chitharagi* (D), Ranjitha S. Gowda, \\ Krishna Karthik and B. Monisha \\ Department of Microbiology, JSS Medical College, JSSAHER, Mysore - 570 015, Karnataka, India.
}

\begin{abstract}
Dengue fever is one of the common viral illnesses linked with significant morbidity and mortality. Of late, there is rapid increase in dengue cases in India. The present study aimed to known dengue seropositivity in different seasons of a year, other laboratory parameters and its geographical distribution. During the study period of 15 months $\mathbf{4 1 7 2}$ patients hospitalized with probable dengue fever were included. Dengue serology was done for all cases by ELISA(J. Mitra \& Co). Demographic details were taken to create geographical maps of the disease. Of the $\mathbf{4 1 7 2}$ suspected dengue cases, 817 had confirmed dengue illness. Of these 817 cases, 584 showed Ns1 antigen (71.4\%), 150 IgM antibody (18.3\%), and 38 had both Ns1 and Ig M (4.6\%). Platelet count less than 1,00,000 were seen in $566(69.27 \%)$ patients. Dengue was noticeably more during monsoon time (June to September) of a year. Through geographical maps major clusters were noticed urban areas of Mysuru and Krishnaraja Nagara, while few clusters were also observed in Periyapatna and T Narsipura taluks of Mysuru district. To minimize the load of dengue cases and its death rate better community awareness and vector control measures need to be strengthened during monsoon especially in areas where burden of the disease is more. This study helps in early preparedness of the authorities concerned in controlling possible epidemics in future.
\end{abstract}

Keywords: Dengue, ELISA, Ig M, NS1, Serology

*Correspondence: vidyavinayakik@gmail.com

(Received: September 21, 2020; accepted: December 28, 2020)

Citation: Rao MR, Mahale RP, Shivappa S, Chitharagi VB, Karthik K, Monisha B. Clinico-Serological Profile and Geographical Distribution of Dengue Fever from a Tertiary Care Hospital, South India. J Pure Appl Microbiol. 2021;15(1):100-104. doi:10.22207/ JPAM.15.1.04

(C) The Author(s) 2021. Open Access. This article is distributed under the terms of the Creative Commons Attribution 4.0 International License which permits unrestricted use, sharing, distribution, and reproduction in any medium, provided you give appropriate credit to the original author(s) and the source, provide a link to the Creative Commons license, and indicate if changes were made. 


\section{INTRODUCTION}

Dengue an Arbo viral disease transmitted by mosquito has rapidly spread across the globe in recent times ${ }^{1}$. The word "Dengue" is derived from Swahili phrase ka-dingapepo means "cramp like seizure" ${ }^{\prime 2}$. According to the WHO estimation, world's two third of the population are at the risk of the disease, especially the tropical and subtropical countries. In the year 1780 , Benjamin Rush in Philadelphia $1^{\text {st }}$ reported dengue and described its condition as "break-borne disease" .

In South East Asian region, India stands as one of the seven countries reporting high incidence of dengue outbreaks that threatens the health care system. Back in 1940's the 1st report of dengue in India was confirmed $\&$ after that more states have been reporting this illness ${ }^{4}$.

Increased occurrence of dengue is due to population growth facilities, lack of vector control, increased urbanization and climatic changes. The Aedes egypti mosquito is responsible mainly for the transmission of dengue fever. The seasonal transmission of dengue is more seen in monsoon and post monsoon period of the year ${ }^{4}$.

Signs and symptoms like abdominal pain, vomiting, mucosal bleeding, hepatomegaly, fluid accumulation, lethargy, thrombocytopenia and decreased hemocrit are warning signs which enables the clinicians to identify the dengue fever and for the effective management of the dengue cases $^{5}$.

Dengue infection accounts for variations in presentation from mild illness to complicated dengue hemorrhagic fever (DHF) and dengue shock syndrome (DSS). When compared to the adults, severity of the disease (DHF) is more in children (90\%) of the age group $\leq 15$ years ${ }^{4}$.

The four distinct serotypes of dengue virus are dengue virus $01,02,03$ and 04 . Lifelong immunity will be provided only if the infection occurs with one serotype of dengue virus (DENV), but the protection against the subsequent infection is partial and transient. So a person can be infected four times in life time, once with each serotype ${ }^{6}$.

For the successful management of dengue outbreaks, the exact clinical presentation, laboratory profile and epidemiological data is crucial ${ }^{1}$. Since long time, several studies has been carried out to study the epidemiological pattern of dengue transmission through clusters formation obtained by geographical mapping of severe dengue cases, which enables in prediction of future outbreaks and in focusing the public health. It is important to understand the development of dengue outbreaks throughout the year, as it helps in the proper utilization of the resources?

Hence, the present study was carried out to determine the dengue positives in different seasons of the year. And also to evaluate the clinical presentation and laboratory profile along with epidemiological pattern of dengue positives of the year through geographical mapping in the central region of Karnataka (Mysuru) in South India

\section{MATERIALS AND METHODS}

\section{Study area and population investigated}

Study involved 4172 dengue suspected hospitalized patients at tertiary care hospital, Mysuru for a period of 15 months (June 2018-August 2019).

\section{Dengue serology}

From serum sample of all the suspected dengue fever patients, Ns1 antigen and IgM antibodies were detected by the Enzyme-linked immunosorbent assay from J.Mitra \& Co.

\section{Geographical Mapping}

- Of the total 4172 patients of suspected dengue illness, a total of 817 patients were confirmed dengue illness either due to the presence of Ns1 antigen or IgM antibodies or both were included in the study.

- Tested negative for NS1 antigen and/or IgM antibody through ELISA were not included in the study.

- And also all the cases beyond the boundary (Mysuru district) were excluded while constructing GIS maps.

- A detailed demographic data was extracted along with corresponding home addresses which indicated the geographic location of the patients. The coordinates obtained using the addresses were only approximations of exact locations.

- Geographical maps indicating the disease were constructed using GIS (Geographic information system) software (version 10.2.2).

- The data containing total number of samples received along with number of positives in each month of the year were taken to 
determine the dengue positivity in different seasons of a year by plotting a bar graph.

\section{Other laboratory investigations}

The laboratory investigations loke total WBC count, PCV and platelet count of each patient was analysed to know their range in dengue illness.

\section{RESULTS}

During the study period of 15 months (June 2018-August 2019), of the total4172 samples, 817 confirmed positive dengue illness through ELISA Of these 817 cases, 584 showed Ns1 antigen (71.4\%), 150 IgM antibody (18.3\%), and 83 had both Ns1 and Ig M (10.15\%) as shown in (Graph 1).

Dengue positivity was considerably high during monsoon period (June to August) of

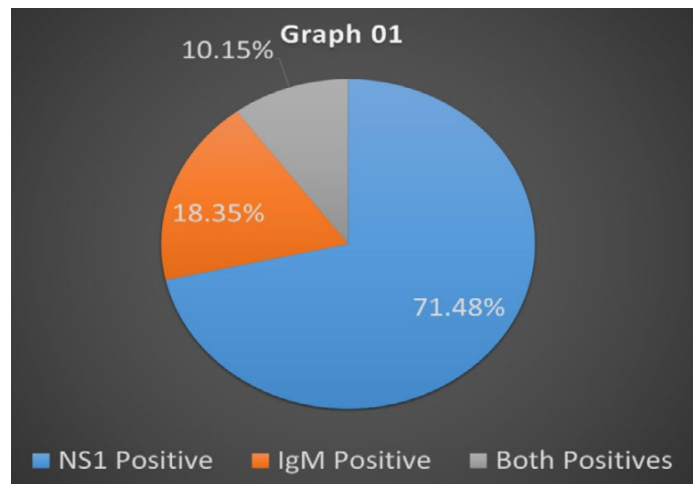

Graph 1. Distribution of dengue seropositivity the year 2018 and 2019 which is represented in (Graph 2). Following mapping of these 817 positive dengue illness cases, majority of the clusters were observed in Krishnarajanagarataluk and urban areas of Mysuru district as shown in (Map 01).

The blood profile of the 817 positive dengue illness patients showed decreased total leukocyte count $(\leq 4,000)$ in $321(39.29 \%)$ patients, increased total leukocyte count $(\geq 11,000)$ in 131 $(16.03 \%)$ patients , increased $(\geq 40 \%)$ packed cell volume in 292 (35.74\%) and platelet count less than 1,00,000 were seen in 566 (69.27\%) patients as shown in (Table 1 ).

\section{DISCUSSION}

In tropical and sub-tropical areas dengue has emerged as an important disease. Rise in dengue cases in resent years is due to unprecedented global population, growth with unplanned and uncontrolled urbanization and also due to poor sanitation facilities in developing nations thereby contributing fertile breeding grounds for mosquitoes ${ }^{8}$.

Table 1. Laboratory parameters

\begin{tabular}{lc}
\hline Lab Parameters & No. of Patients (817) \\
\hline $\operatorname{TLC}(\leq 4,000)$ & $321(39.29 \%)$ \\
$\operatorname{TLC}(\geq 11,000)$ & $131(16.03 \%)$ \\
$\operatorname{PCV}(\geq 40 \%)$ & $292(35.74 \%)$ \\
Platelet $(\leq 1,00,000)$ & $566(69.27 \%)$ \\
\hline
\end{tabular}

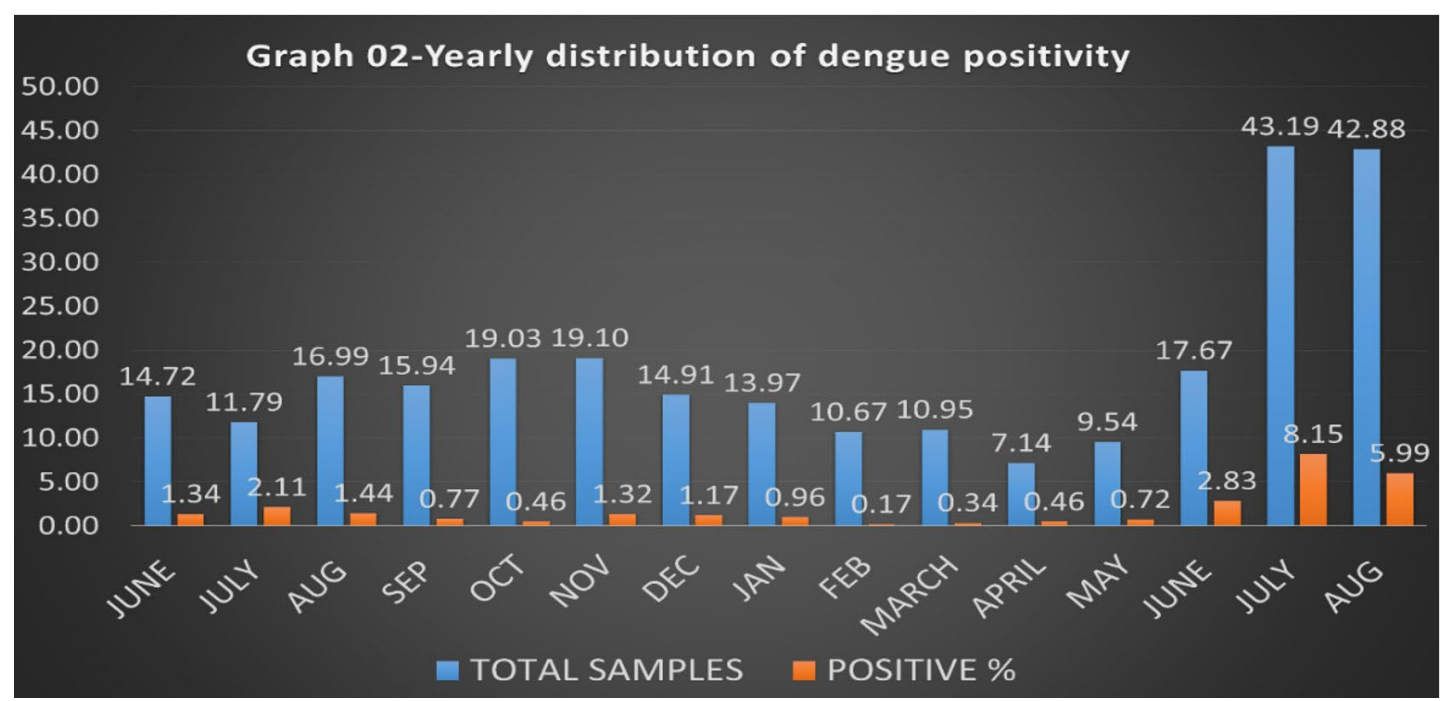

Graph 2. Yearly distribution of dengue positivity. 


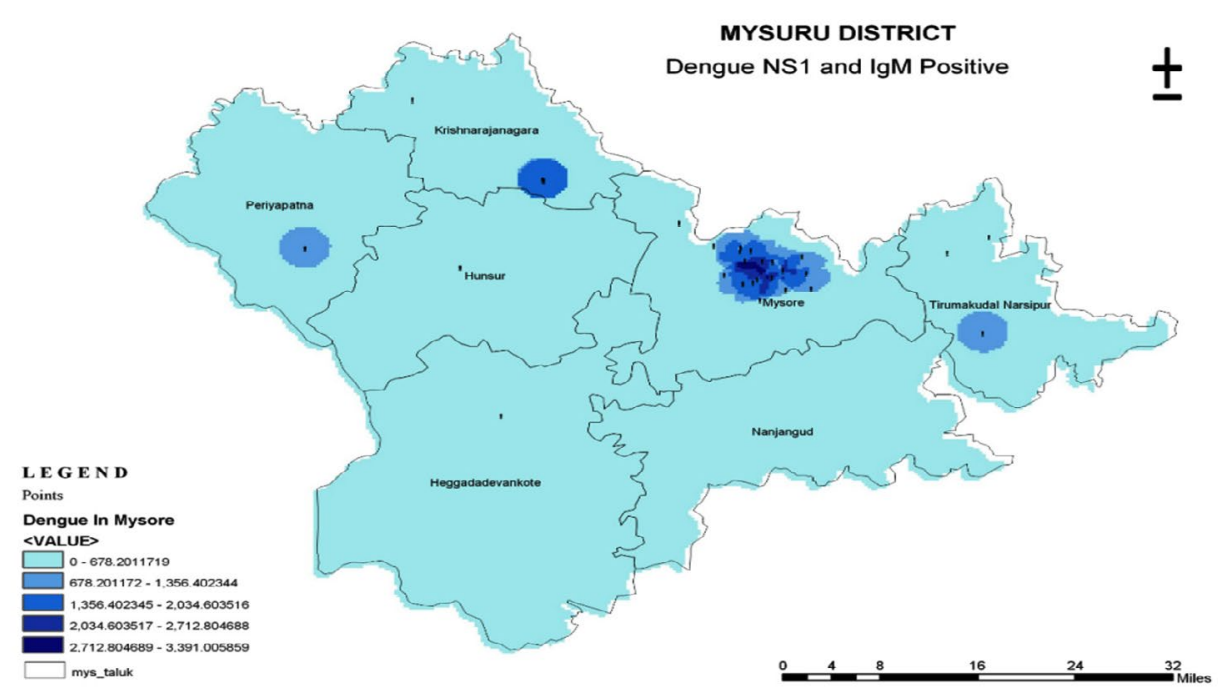

Map 1. Geographical Representation of Dengue Positivity in Mysuru District.

This study describes the clinical profile, laboratory features and geographical distribution of dengue infection in a tertiary care hospital of South India.

In the present study patient admissions were maximum during the rainy season (i.e. august to October) that provides favorable conditions for the growth of vector Aedes egypti, similar observations were reported in the study conducted by Lakshmi and Rajendra?

In our study NS1 antigen positive patients were more in number (71.4\%) when compared to IgM antibody positives and/or both NS1 and IgM positive patients. A similar study conducted by Mohamed et al., 2014 showed alike results $(76.7 \%)^{2}$, remarking no significant difference in both the study.

Our study showed leukopenia in $39.29 \%$ patients and Leukocytosis in $16.03 \%$ patients whereas in the study conducted by Katari et al., $2019^{(1)}$ showed leukopenia in $30.2 \%$ patients and leukocytosis in $8.1 \%$ patients respectively, which showed significant differences in both the studies remarking that more number of patients in our study are encountered with both leukopenia and leukocytosis.

In our study, platelet count $\leq 1,00,000$ was seen in $69.27 \%$ patients but in the study conducted by Katari et al., $2019^{1}$ and Adarsh and
Subramanian, $2015^{(3)}$, platelet count $\leq 1,00,000$ was seen in $89.46 \%$ and $86.29 \%$ patients respectively, remarking that in our study not more number of patients were encountered with thrombocytopenia.

Similarly in our study increased packed cell volume was seen in $35.74 \%$ patients but in the study conducted by Katari et al., 2019 ${ }^{1}$ and Mohamed et al., 2014 ${ }^{2}$ showed increased packed cell volume in $67.59 \%$ and $57.53 \%$ patients showing significant differences in both the studies remarking that only less number of patients showed raised hematocrit in our study.

In our study, through geographical mapping majority of the clusters of dengue illness positives were seen in Krishna raja Nagara and urban areas of Mysuru, which was similar to the study conducted by Indrajit, $2013^{10}$ therefore remarking that there could be an outbreak of dengue cases between the months of August to October as it constitutes $55 \%$ of the total rainfall of the year, which provides favorable conditions for the growth of vector Aedes aegypti.

Other major reasons for the outbreak of dengue illness in Mysuru district includes, infrastructure development and building projects in the district being increased in the last 5 years. Mysuru district being an important tourist destination is frequented by travelers from within 
India and abroad which facilitates transport activities and travelers could themselves act as a mode of introduction of different viral strains. And also Mysuru district has large fertile lands for cultivation of paddy and pulses and there is high usage of nitrogenous fertilizers in farming which contaminates the ground water rendering them rich in nitrates. Similarly depletion of water resources in non-monsoon period of the year facilitates increased storage of deep ground water pumped from bore wells which is rich in nitrates that serve as an excellent and attractive vector habitats as described by Indrajit, $2013^{10}$.

\section{CONCLUSION}

Dengue is one of the emerging infectious disease in the recent years and Mysuru district experiences yearly and regular outbreaks of dengue. Our study highlights the clinical and geographical pattern of presentation of the disease in correlation with the laboratory parameters and the disease outcomes. It can be concluded that the clinical presentation of dengue illness varies considerably from place to place. In order to minimize the load of dengue cases and its death rate, increased community awareness and vector control measures need to be strengthened during monsoon period, especially in urban areas Mysuru district where there was an outbreak.

\section{ACKNOWLEDGMENTS}

None.

\section{CONFLICT OF INTEREST}

The authors declare that there is no conflict of interest.

\section{AUTHORS' CONTRIBUTION}

All authors listed have made a substantial, direct and intellectual contribution to the work, and approved it for publication.

\section{FUNDING}

None.

\section{DATA AVAILABILITY}

All datasets generated or analyzed during this study are included in the manuscript.

\section{ETHICS STATEMENT}

This article does not contain any studies with human participants or animals performed by any of the authors.

\section{REFERENCES}

1. Katari S, Dorasanamm M, Nagabhushana MV. The clinical and laboratory profile of dengue fever in a tertiary care hospital. International Journal of Advances in Medicine. 2019;6(5):1447-1451. doi: 10.18203/2349-3933.ijam20193625

2. Mohamed MK, Kalavathi GP, Mehul R, et al. A Study of Clinical and Laboratory Profile of Dengue Fever in Tertiary Care Hospital in Central Karnataka, India. Global Journals Inc. (USA). 2014;14(5):2249-4618.

3. Adarsh E, Subramanian V. Clinical profile of dengue infection in a tertiary care hospital. Indian Journal of Child Health. 2015;2(2):68-71.

4. Gupta N, Srivastava S, Jain A, Chaturvedi UC. Dengue in India. Indian J Med Res. 2012;136(3):373-390.

5. Changa K, Fredrico D, Diana LTT, David CL. When less is more: can we abandon prophylactic platelet transfusion in Dengue fever? Annals of the Academy of Medicine of Singapore. 2011;40:539-545.

6. Karoli R, Fatima J, Siddiqi Z, Kazmi KI, Sultania AR. Clinical profile of dengue infection at a teaching hospital in North India. J Infect Dev Ctries. 2012;6(07):551-554. doi: $10.3855 /$ jidc. 2010

7. Gary KK, Papapreponis P, Isa RM. Geographical distribution and spatio-temporal patterns of hospitalization due to dengue infection at a leading specialist hospital in Malaysia. Geospatial Health. 2018;13(1):642. doi: 10.4081/gh.2018.642

8. Gubler DJ. Dengue and Dengue Hemorrhagic Fever. Clin Microbiol Rev. 1998;11(3),480-496. doi: 10.1128/ CMR.11.3.480

9. Lakshmi DV, Rajendra PS. Clinical Profile of Dengue Fever in a Tertiary Care Hospital Hyderabad. Global Journal of Medical Research: F Diseases. 2018;18(1):2249-4618.

10. Indrajit S. Pediatric dengue trends observed in a tertiary care hospital of south India during 2013. doi: 10.13140/2.1.2843.4565. 